\title{
Interação de Usuários de Alto e Baixo Letramento: Um Estudo de Caso utilizando o Eyetracking
}

\author{
Ney Wagner F. Cavalcante ${ }^{1}$, Letícia Régis Di Maio ${ }^{1}$, Simone Bacellar Leal \\ Ferreira $^{1}$, José Luiz dos Anjos Rosa ${ }^{2}$, Hélio da Silva Braga ${ }^{1}$ \\ ${ }^{1}$ Departamento de Informática - Universidade Federal do Estado do Rio de Janeiro - \\ UNIRIO \\ Rio de Janeiro - Brazil \\ ${ }^{2}$ Centro Universitário Estadual da Zona Oeste- UEZO \\ Rio de Janeiro - Brazil \\ \{ney.cavalcante, leticia.maio, simone, helio.braga\}@uniriotec.br, \\ jrosa@uezo.rj.gov.br
}

\begin{abstract}
The information technology including, increasingly, the services offered to citizens, this necessitates the development of web pages accessible to everyone, regardless of education level. Whereas a significant portion of the brazilian population is within the low literacy profile, the objective of this research was to analyze the different forms of navigation among users of high and low literacy. Data were collected through user testing through eye tracking. The experiences of interaction were performed from two tasks initiated in the Google search engine and completed in two popular sites. At the end, some suggestions were proposed interface improvements...
\end{abstract}

Resumo. A informatização abrange, cada vez mais, os serviços oferecidos aos cidadãos, isto torna imprescindível o desenvolvimento de páginas web acessiveis para todos, independente do grau de escolaridade. Considerando que uma significativa parcela da população brasileira está dentro do perfil de baixo letramento, o objetivo desta pesquisa foi analisar as formas distintas de navegação entre os usuários de alto e baixo letramento. Os dados foram colhidos através de testes com usuários, por meio de rastreamento ocular. As experiências de interação foram realizadas a partir de duas tarefas iniciadas no motor de busca Google e concluidas em dois sites populares. Ao final, foram propostas algumas sugestões de melhorias de interface.

\section{Introdução}

O uso frequente de tecnologia está crescendo em todo o mundo. Atualmente, há uma migração gradual de rotinas diárias para o meio eletrônico, uma vez que as ferramentas da Web tornaram-se parte da vida cotidiana [Nielsen \& Loranger 2007] desempenhando um papel social, especialmente para usuários com deficiências [Kodagoda \& Wong 2008].

O acesso à informação deve ser facilitado e garantido, uma vez que essa migração traz novos desafios aos pesquisadores e desenvolvedores de sistemas, pois suas interfaces precisam ser acessíveis a todos os perfis de usuários. Para ajudar os 
usuários a recuperar o conteúdo da web, os motores de busca são frequentemente utilizados: $80 \%$ do acesso a páginas da Web vêm dessas ferramentas [Thurow e Musica 2009]. Pesquisar envolve a análise de diferentes tipos de mídia, por isso é uma atividade desgastante mentalmente que exige foco e atenção [Hearst 2009; Modesto et al 2013]. Porém há usuários que possuem limitações relacionadas à alfabetização que podem comprometer a interação principalmente porque o conteúdo disponível na Web é mais textual [Kodagoda 2008]. A maioria dos problemas de usabilidade na Web está relacionada a encontrar, ler e compreender a informação [Nielsen \& Loranger 2007]. Pessoas com baixos níveis de habilidade de leitura têm esses problemas ampliados devido à falta de competências linguísticas, sendo caracterizada também como uma questão de acessibilidade. A falta dessas habilidades afeta a maneira como as pessoas interagem com interfaces de computador, como os motores de busca [Kodagoda et al 2009; Gupta \& Rose, 2010], usados para recuperar o conteúdo da Web , que é predominantemente textual [Hearst 2009].

Os usuários com baixos níveis de competências de leitura têm peculiaridades que devem ser consideradas no projeto e desenvolvimento de sites, tais como limitações de percepção de conteúdo e estratégias de busca [Kodagoda et al 2009 ; Gupta \& Rosa , 2010]. Sendo assim, eles usam a web de uma maneira diferente daqueles usuários que têm altas habilidades de leitura [Kodagoda \& Wong, 2008; Capra et al 2012 ; Modesto \& Ferreira 2013]. O objetivo desta pesquisa foi observar e analisar os usuários, com dois perfis diferentes (usuários de alto e de baixo letramento), identificando suas experiências e detalhes da interação. Isso foi feito durante as formulações de consulta no motor de busca, Google, e a informação desejada a partir de dois diferentes sites comerciais populares.

A Organização das Nações Unidas para a Educação, Ciência e Cultura (UNESCO) [Modesto et al 2013] considera como analfabetos funcionais, pessoas com deficiência nas habilidades de leitura, escrita, cálculo e ciências, cujo nível de escolaridade é equivalente a menos de quatro anos completos de estudo. Essa classificação, adotada pelo Instituto Nacional de Estudos e Pesquisas Educacionais Anísio Teixeira (INEP) e do Instituto Brasileiro de Geografia e Estatística (IBGE) [UNESCO 2012] foi utilizada no estudo. De acordo com a agência brasileira, a taxa de analfabetismo funcional no ano de 2013 no Brasil foi estimada em 20\% do total.

Para observar as interações de usuários, adotou-se o método de rastreamento ocular para coletar dados. Foi utilizado o Tobii T120, rastreador ocular de Tobii Tecnologia [Tobii 2013]. Para investigar a relevância entre texto e imagem nas informações disponíveis nos sites analisados, foi utilizado o padrão de movimentos sacádicos e fixações. Para a execução das tarefas, foram observados dez usuários, cinco para cada perfil de letramento. Este número foi definido tendo em conta as recomendações propostas por Nielsen [Nielsen 2000]. À medida que o número de usuários aumenta, a informação que é recolhida tende a se repetir, proporcionando uma menor quantidade de informações novas. Segundo a pesquisa, cinco usuários são capazes de detectar 85\% dos problemas de usabilidade [Nielsen 2000]. Para selecionar e definir o perfil dos usuários, os voluntários responderam a um questionário.

Durante os testes, os participantes fizeram duas consultas em um motor de busca (Google) e realizaram as tarefas determinadas em dois sites comerciais diferentes, 
selecionados com base na familiaridade com as suas lojas físicas. O tempo para completar as tarefas foi estabelecido entre os pesquisadores, mas desconhecido pelos usuários buscando naturalidade durante a interação. Além do rastreamento ocular, foram adotados os métodos de observação direta (em que os pesquisadores observam o caminho seguido pelos usuários durante a navegação) e o protocolo de verbalização consecutiva. Os testes foram realizados em ambiente de laboratório controlado. Após responder ao questionário e assinar um termo de consentimento, o equipamento rastreador ocular foi apresentado a cada participante. Após a calibração de rastreamento do olhar, as tarefas impressas em folhas foram oferecidas aos usuários. Ao final de cada tarefa, um breve questionário foi preenchido por cada usuário para verificar as dificuldades em torno da execução da mesma, além de sugestões de melhorias de interface.

\section{Alfabetização no Brasil}

Alfabetização pode ser analisada por diferentes perspectivas e conceituada de diferentes maneiras. Um entendimento comum sobre a alfabetização envolve vocabulário, leitura e escrita, além de habilidades como lógica, matemática, análise simbólica (imagens e sons) e interpretação de texto [UNESCO 2012]. Na verdade, o conceito de analfabetismo funcional varia de região para região. Hoje em dia é uma abordagem comum para considerar que essas habilidades devem ser contextualizadas e que não são desenvolvidas igualmente entre indivíduos diferentes. Além disso, conceito de alfabetização também considera os aspectos funcionais, que significam capacidade de aplicação de vocabulário, leitura e escrita em diferentes áreas da vida diária, como na computação, ecologia , saúde e outras áreas [UNESCO 2012].

A Organização das Nações Unidas para Educação, Ciência e Cultura (UNESCO) [Modesto et al 2013] considera pessoas como analfabetos funcionais entre 15 e 64 anos de idade, que não têm o domínio de habilidades em leitura, escrita , cálculos e ciências, o que corresponde a uma educação de menos de quatro anos de estudo [Capra et al 2011; Modesto \& Ferreira 2013]. Essa classificação, adotada pelo Instituto Nacional de Estudos e Pesquisas Educacionais Anísio Teixeira (INEP) e do Instituto Brasileiro de Geografia e Estatística (IBGE) [UNESCO 2012] foi utilizada no presente trabalho. Uma pesquisa realizada pelo IBGE mostrou que 29 milhões de pessoas no Brasil são analfabetos funcionais, que significa mais de $20 \%$ da população total [Modesto et al, 2013]. Neste trabalho, esse conceito foi adotado, mas os participantes foram chamados de "usuários de baixo letramento", uma vez que um dos protocolos utilizados para orientar o estudo recomendou não chamá-los de analfabetos funcionais [Capra et al, 2012].

Existem outros critérios que poderiam ser utilizados no estudo, em vez de considerar apenas a idade e os anos de estudo na educação formal. Há instituições que desenvolveram testes de alfabetização e numéricos específicos, com níveis de dificuldade e pontuação, para avaliar as habilidades das pessoas em leitura, escrita, cálculos e ciências. No entanto, estes testes são privados, então não há ferramentas públicas que nos permitem classificar os usuários desta forma [Capra et al 2012]. 


\subsection{Eyetracking e testes com usuários}

Eyetracking é a tecnologia que permite fazer o rastreamento dos movimentos oculares do observador através de raios infravermelhos, registrando o percurso percorrido pelo olhar, assim como ponto de atenção [Barboza 2011]. Uma vez que rastreia o caminho percorrido pelos olhos do usuário, pode apoiar o desenvolvimento e aperfeiçoamento de interfaces, que, se bem projetadas, podem tornar-se uma fonte de motivação ou um fator decisivo para a rejeição do sistema em caso contrário [Ferreira \& Nunes 2008].

No contexto da web, é importante considerar os analfabetos funcionais como usuários potenciais e propor soluções tecnológicas acessíveis para esse público, considerando as diretrizes de acessibilidade [Melo et al 2009]. Uma das dificuldades dos usuários com baixa escolaridade é a compreensão de um texto. Chama-se inteligibilidade a característica do texto que faz com que ele seja fácil de ser lido e compreendido. Textos fáceis podem compensar os baixos níveis de conhecimento prévio, habilidade de leitura, interesse e motivação [Barboza 2011]. Logo, as soluções propostas devem ser adequadas a um público mais letrado, mas devem garantir que um texto seja facilmente lido e compreendido pelos analfabetos funcionais [Capra et al 2012].

\subsection{Trabalhos relacionados}

Em sua pesquisa, Barboza [Barboza 2011] fez um interessante levantamento bibliográfico sobre usabilidade e linguagem clara em websites governamentais com usuários de alto e baixo letramento e menciona o uso do eyetracking. No entanto, não aplicou testes com os mesmos.

Em seu trabalho, Lukasova [Lukasova 2012] utilizou o eyetracking em conjunto com testes de Ressonância Magnética Funcional para investigar mudanças de comportamento e funcionamento neural em crianças e adultos com o objetivo de contribuir para estudos futuros voltados para grupos clínicos específicos, tais como dislexia do desenvolvimento, autismo e esquizofrenia. Apesar da relevância do trabalho, a autora não utilizou adultos com nenhum tipo de deficiência ou dificuldade de aprendizado.

\section{Método de pesquisa}

A presente pesquisa, de caráter exploratório, foi baseada no método qualitativo de coleta de dados consistindo em quatro etapas, descritas a seguir:

a. Seleção do perfil dos usuários que participaram da pesquisa: decidiu-se escolher dez adultos, formando dois grupos distintos. O primeiro grupo composto de cinco adultos com escolaridade de até três séries completas de ensino, ou seja, menos de quatro anos de estudos completos de acordo com a classificação da UNESCO [UNESCO 2012]. O segundo grupo, composto de cinco adultos alfabetizados plenamente e com cinco anos de estudo ou mais. Todos os participantes possuem conhecimentos básicos em navegação na web de no mínimo três meses e possuem idades de 18 a 64 anos.

b. Seleção dos sites e definição de tarefas: foram escolhidos dois sites comerciais de lojas brasileiras populares, de acordo com o perfil dos usuários. Na primeira loja, de material de construção, "Casa Show", o usuário deveria ver o preço de um determinado 
item. Na segunda loja, de venda de óculos, "Óticas do Povo", o usuário deveria encontrar o endereço de uma determinada loja física.

c. Estudo de Caso: foi feito um estudo de caso (detalhado na seção 4) com os dois grupos, executando as tarefas determinadas.

d. Análise dos dados: A tecnologia do eyetracking gera resultados complementares aos testes tradicionais de usabilidade, com relatórios sobre a navegação dos usuários [TOBII, 2013]. Os dados oriundos desse tipo de teste apresentam formatos variados (quantitativos, estatísticos, de imagem e de vídeo), sinalizando o caminho percorrido durante a navegação, de forma sequencial e com indicativos de duração em cada ponto de fixação dos olhos do participante, podendo detectar onde houve maior esforço cognitivo e prováveis problemas de usabilidade não explorados. Os resultados dos testes com o uso do eyetracking estão apresentados na seção 5 de Análise de dados.

\subsection{Limitações}

Realizar observações com a participação de usuários não é fácil; dentre as complexidades desse processo encontra-se a dificuldade de obter voluntários com disponibilidade para realizar os testes, representando um obstáculo na acessibilização completa dos sistemas [Ferreira et al. 2007].

Foi necessário categorizar os usuários e sendo assim, foi escolhida a classificação da UNESCO, que é adotada pelo Instituto Brasileiro de Geografia e Estatística (IBGE). Se fossem utilizados outros testes para avaliar as habilidades de leitura e escrita dos usuários, outros resultados poderiam ter sido obtidos. Apesar da grande variedade de idades adotada como critério, foram selecionados os usuários que tinham o mesmo nível de educação e experiência semelhante com computadores, a fim de minimizar um possível viés causado pela idade.

\section{Estudo de caso}

Preparação do ambiente de testes: Inicialmente os pesquisadores definiram os perfis dos usuários e classificaram em dois grupos distintos, com cinco usuários cada, sendo o primeiro formado por indivíduos de baixo letramento e o segundo de alto letramento. Em seguida, foram enviados convites para participação da pesquisa de acordo com o perfil educacional dos usuários para associações, universidades e centros comunitários. Onze usuários aceitaram, sendo um deles selecionado para realizar o teste piloto.

Operacionalização dos testes: Foi aplicado um teste piloto, composto por duas tarefas em dois sites comerciais de lojas populares brasileiras, com um usuário antes de realizar o teste com os dez usuários restantes. Todos assinaram um termo de consentimento para participarem da pesquisa que foi lido em voz alta pelos pesquisadores, além de terem sido fornecidos esclarecimentos sobre a pesquisa. Após isso, os participantes responderam a um questionário impresso que foi utilizado para a classificação do perfil, de acordo com a escolaridade declarada.

Em seguida, o equipamento Eyetracking foi apresentado e cada participante teve os olhos calibrados pelo aparelho, além de receberem as devidas orientações sobre postura, concentração para evitar possíveis perdas de registros pelo rastreador. Explicou-se a quantidade de tarefas exigidas no teste, bem como a liberdade e a esperada autonomia que o usuário teria para a execução das mesmas, antes de qualquer solicitação de ajuda 
aos pesquisadores. Para garantir o anonimato dos participantes, seus nomes foram codificados da seguinte forma: LL_01, LL_02, LL_03, LL_04 e LL_05 para o grupo com baixo letramento e HL_01, HL_02, HL_03, HL_04 e HL_05, para usuários com alto letramento. A tabela 1 apresenta o perfil de todos os participantes e o tempo total de execução de cada tarefa.

Com o objetivo de melhor compreender o conteúdo lógico e estratégico que levou cada usuário a tomar uma decisão durante a realização de uma tarefa, seja por um erro, desvio ou distração, solicitou-se que os participantes verbalizassem seus pensamentos após a sessão de avaliação, ou seja, adotou-se o protocolo de verbalização consecutiva. Não se optou pela verbalização simultânea, pois de acordo com "Protocolos de Avaliação de Acessibilidade Web com Analfabetos Funcionais", proposto por Capra (Capra, 2011) e (Capra, 2012), a verbalização simultânea é uma barreira para usuários de baixo letramento.

Tabela 1: Perfil dos participantes

\begin{tabular}{|c|c|c|c|c|c|c|c|}
\hline \multicolumn{8}{|c|}{ Dados básicos dos usuários } \\
\hline Usuário & Sexo & Idade & Formação & $\begin{array}{c}\text { Computador } \\
\text { próprio }\end{array}$ & $\begin{array}{c}\text { Frequência } \\
\text { semanal na } \\
\text { Internet }\end{array}$ & $\begin{array}{c}\text { Tempo } \\
\text { execução } \\
\text { tarefa } 1\end{array}$ & $\begin{array}{c}\text { Tempo } \\
\text { execução } \\
\text { tarefa } 2\end{array}$ \\
\hline LL_01 & $\mathrm{F}$ & 59 & $\begin{array}{l}3 \text { anos Ensino } \\
\text { Fundamental }\end{array}$ & $\operatorname{Sim}$ & 01 & $06 \mathrm{~min}$ e $50 \mathrm{~s}$ & $05 \mathrm{~min}$ e $22 \mathrm{~s}$ \\
\hline LL_02 & M & 64 & $\begin{array}{c}3 \text { anos Ensino } \\
\text { Fundamental }\end{array}$ & Sim & 03 & $04 \mathrm{~min}$ e $05 \mathrm{~s}$ & $10 \mathrm{~min}$ e $03 \mathrm{~s}$ \\
\hline LL_03 & $\mathrm{F}$ & 58 & $\begin{array}{c}3 \text { anos Ensino } \\
\text { Fundamental }\end{array}$ & Sim & 01 & $02 \mathrm{~min}$ e $42 \mathrm{~s}$ & $06 \min$ e $55 \mathrm{~s}$ \\
\hline LL_04 & M & 62 & $\begin{array}{l}3 \text { anos Ensino } \\
\text { Fundamental }\end{array}$ & Sim & 01 & $02 \mathrm{~min}$ e $22 \mathrm{~s}$ & $06 \min$ e $49 s$ \\
\hline LL_05 & M & 59 & $\begin{array}{l}3 \text { anos Ensino } \\
\text { Fundamental }\end{array}$ & $\operatorname{Sim}$ & 01 & $05 \min$ e $31 \mathrm{~s}$ & $05 \min$ e $26 s$ \\
\hline HL 01 & M & 36 & Doutorado & Sim & 07 & $01 \min$ e $49 \mathrm{~s}$ & $34 \mathrm{~s}$ \\
\hline HL_02 & M & 35 & Especialização & Sim & 07 & $01 \mathrm{~min}$ e $02 \mathrm{~s}$ & $36 \mathrm{~s}$ \\
\hline HL_03 & $\mathrm{F}$ & 38 & Doutorado & Sim & 07 & $51 \mathrm{~s}$ & $36 \mathrm{~s}$ \\
\hline HL_04 & M & 40 & Doutorado & Sim & 07 & $57 \mathrm{~s}$ & $44 \mathrm{~s}$ \\
\hline HL_05 & M & 33 & Doutorado & Sim & 07 & $46 s$ & $39 \mathrm{~s}$ \\
\hline
\end{tabular}

Aplicação dos testes: Após os ajustes iniciais, iniciaram-se os testes, que continham as mesmas tarefas do teste piloto, com os 10 usuários. Um vídeo dos testes foi gravado pelo rastreador e em paralelo os pesquisadores fizeram anotações utilizando a técnica de observação direta, simultaneamente às gravações do rastreamento. Foram dadas as orientações sobre cada tarefa e emissão de comentários positivos para estimular o 
usuário a continuar o teste. Cada tarefa deveria ser concluída em até dez minutos, porém os pesquisadores só poderiam oferecer ajuda após os cinco primeiros minutos.

Após o término de cada tarefa, ocorreu o preenchimento de um questionário sobre a realização das mesmas e uma rápida entrevista com o usuário sobre o processo de interação com os sites e as funcionalidades das tarefas.

Com os relatórios gerados foi feito um levantamento de dados utilizando algumas métricas coletadas durante a navegação dos participantes para posterior comparação com os relatórios gerados pelo eyetracking, modelo TOBII T120.

O equipamento de rastreamento ocular pode gerar os mais variados tipos de relatórios de acordo com as métricas desejadas pelos pesquisadores. Por conta disso, após os testes foram determinadas as Áreas de Interesse (AOI) que seriam relevantes para a pesquisa. Em seguida, foram extraídos os dados quantitativos, de imagem e de vídeo de cada participante que mostram o caminho percorrido durante a navegação, além de captar o comportamento do usuário.

\section{Análise dos dados}

Para a análise de dados, foram adotadas as métricas do rastreamento ocular: First Fixation Duration (FFD) duração em segundos da primeira fixação em uma AOI; Fixation Count (FC) número de vezes que o participante se fixa em uma AOI; Mouse Click Count (MCC) número de cliques do mouse de um participante em uma AOI; Time To First Fixation (TTFF) tempo, desde o início do estímulo, até a primeira fixação em uma AOI e Time To First Mouse Click (TTFMC), tempo até o primeiro clique do mouse de um participante em uma AOI.

As áreas de interesse foram escolhidas a partir recurso de mapa de calor, que registrou as áreas que chamaram mais a atenção dos participantes, bem como os elementos de interface que, receberiam interação dos usuários para que concluíssem as tarefas. Foram delimitadas as áreas de interesse: "botão de pesquisa Google"; "botão inferior de pesquisa Google”; "campo de pesquisa Google em página interna”; "campo de pesquisa Google”; "campo superior de pesquisa Google”; "link para página inicial do site"; "primeiro link patrocinado Google"; "sugestão de pesquisa da área de texto"; "campo de pesquisa Casa Show"; "menu hidráulica Casa Show”; "imagem caixa d'água pequena”; "imagem caixa d'água grande”; "menu lojas Óticas do Povo”; "banner central Óticas do Povo"; "banner inferior Óticas do Povo"; "mapa imagem Óticas do Povo"; "mapa texto Óticas do Povo"; "endereço loja Óticas do Povo" e "Ctrl + F Óticas do Povo

\subsection{Observações feitas durante a $1^{\mathrm{a}}$ tarefa}

Na primeira tarefa os usuários deveriam partir do site do Google, localizar o site da loja "Casa Show" para consultar o preço da caixa d'água da marca Fortlev, de 1000 litros. O tempo de conclusão da $1^{\text {a }}$ tarefa foi de 258 segundos, em média, para os usuários de baixo letramento, enquanto que os de alto letramento demoraram cerca de 65 segundos.

$1^{\circ}$ Passo (Busca no site Google): Associando a métrica FC (Fixation Count) com a área de interesse (AOI) "campo de pesquisa Google", observou-se que, antes de digitarem, nove usuários clicaram no campo de pesquisa do buscador e apenas um (LL_01) clicou 
no botão "Pesquisa Google". Este usuário, durante a verbalização consecutiva, comentou que precisou "ligar" o Google clicando no botão, antes de pesquisar.

A partir da associação da métrica TTFMC (Time To First Mouse Click) com a área de interesse (AOI), "campo de pesquisa Google", pode-se afirmar que em média, os usuários de baixo letramento, demoraram 38,8 segundos para clicar neste campo enquanto que os de alto letramento gastaram cerca de 3,6 segundos.

A partir da associação da métrica MCC (Mouse Click Count) com a AOI "botão de pesquisa Google", observou-se que, com exceção do primeiro usuário de baixo letramento, todos participantes desconsideraram o botão de pesquisa do Google, optando pelo uso da tecla ENTER.

O recurso de barra de rolagem foi utilizado pela totalidade dos usuários de baixo letramento, ao contrário do outro grupo que preferiu usar o atalho do teclado $(\mathrm{Ctrl}+\mathrm{F})$, ou os campos de busca dos sites.

A partir da associação das métricas FC (Fixation Count) e MCC (Mouse Click Count) com a AOI "sugestão de autopreenchimento de pesquisa do Google", foi possível observar que entre os três usuários de alto letramento que leram as sugestões do Google, apenas um aproveitou esse recurso. Em contrapartida, todos usuários de baixo letramento ignoraram as sugestões. Esse comportamento já havia sido observado na pesquisa. [Capra 2011] afirma que usuários de baixo letramento possuem comportamentos diferentes de usuários com alto letramento. $\mathrm{O}$ contexto do usuário também influencia seus modelos mentais, como seu país, cultura, língua, grau de instrução, idade e experiência com computadores [Ferreira \& Nunes 2008]. Usuários com menos conhecimento são menos flexíveis em suas estratégias de busca e tendem a não recorrer a novas abordagens [Modesto 2012].

A partir da associação das métricas FFD (First Fixation Duration) e TTFMC (Time To First Mouse Click) com as AOIs "link para página inicial do site da tarefa" e "link patrocinado do Google", foi possível observar que todos os usuários de baixo letramento se confundiram com os links patrocinados para sites concorrentes, em detrimento à atenção que deveriam ter aos links das páginas iniciais do site proposto nesta tarefa. Sendo assim, todos necessitaram reiniciar a tarefa. A tendência de abandonar a busca também foi percebida quando se dão por satisfeitos com suas pesquisas, ainda que não tenham obtido o melhor ou mais correto resultado, o que coincide com as pesquisas de Modesto [Modesto 2012].

Já a maioria dos usuários de alto letramento desprezou os links patrocinados, tendo sido muito mais atentos aos objetivos propostos nas tarefas.

O site do motor de busca Google apresenta resultados de pesquisa em duas linhas: a primeira como link (sublinhada) reporta o usuário à outra página e a segunda (posicionada abaixo do link) como URL do site a ser visitado. Sabendo que o link é composto de palavras-chave da pesquisa feita pelo usuário e que, nem sempre a URL associada ao link corresponde ao endereço esperado pelo usuário, percebeu-se que o participante de baixo letramento se confunde com essa análise de resultados.

Como os participantes de baixo letramento possuem dificuldade de transferir sua atenção rapidamente de um assunto para outro sem se perderem, no momento em que digitavam, eles só conseguiam manter o foco no campo de entrada de texto ou no 
teclado. O recurso não influenciou nas buscas quando se tratou de prover feedback mais rápido ao usuário [Modesto 2012]. Essa observação pode ser confirmada a partir do caminho percorrido pelo olhar (gaze plot) que mostra ter havido mais fixações nos links, do que nas URL's (posicionadas abaixo de cada link do resultado de buscas).

$2^{\mathbf{0}}$ Passo (Busca no site Casa Show): A página inicial do site "Casa Show" foi visitada por oito participantes, sendo quatro de cada perfil, comprovado pela métrica TTFF (Time To First Fixation) na AOI "menu hidráulica Casa Show", sendo que os comportamentos de cada perfil de participantes foram distintos. Os de baixo letramento navegaram a partir do menu vertical do site com o auxílio da barra de rolagem, mas apenas um reconheceu que o subitem de menu contendo a opção "Hidráulica" levaria para o produto "caixa d'água" proposto na tarefa, o que pode ser comprovado pela métrica FC (Fixation Count) com a AOI acima descrita.

A partir da métrica TTFC (Time To First Fixation) com a AOI “imagem caixa d'água pequena Casa Show" foi observado que, dentro do perfil de baixo letramento, três usuários cumpriram a tarefa por meio de fixação da imagem, ao contrário dos outros dois, que cumpriram a tarefa pela análise textual, uma vez que o valor dessa métrica (TTFF) sobre a área de interesse acima, resultou em zero.

Dos quatro usuários de alto letramento que navegaram na página inicial, apenas um localizou a página interna com o produto clicando na opção "Hidráulica", de acordo com a associação entre a métrica MCC (Mouse Click Count) e a área de interesse (AOI) "menu hidráulica Casa Show". A análise da métrica MCC (Mouse Click Count) e a AOI "campo de pesquisa Casa Show" permitiu observar que dois participantes desse perfil encontraram a página interna com auxílio do campo de pesquisa do site Casa Show. O outro usuário de alto letramento se guiou pelas imagens do site, de acordo com obtido pela associação entre a métrica MCC (Mouse Click Count) e a área de interesse (AOI) "imagem caixa d'água pequena".

Através da associação entre a métrica FC (Fixation Count) e as AOIs "imagem caixa d'água pequena" e "imagem caixa d'água grande" é possível afirmar que os usuários de baixo letramento, antes de interagir com as imagens relativas ao produto pesquisado, tiveram três vezes mais fixações nos textos do que os usuários de alto letramento. Para cumprir a tarefa, os usuários de baixo letramento realizaram uma leitura demorada da maior parte das informações textuais e, só depois, compararam com as imagens para interagir com a interface. Já os participantes de alto letramento navegaram de forma mais objetiva e, talvez em função da maior experiência com a Internet, usaram recursos de otimização de busca, desconsiderando, muitas vezes os menus e as imagens.

\subsection{Observações feitas na $2^{\mathrm{a}}$ tarefa}

Na segunda tarefa, os participantes deveriam partir do site do Google e localizar o site das Óticas do Povo para então acharem o endereço da loja do bairro de Campo Grande. O tempo médio de conclusão da $2^{\mathrm{a}}$ tarefa foi de 415 segundos para os usuários de baixo letramento e 38 segundos para os de alto letramento, ou seja, estes últimos foram em média 11 vezes mais rápidos.

$1^{\mathbf{0}}$ Passo (Busca no site Google): A partir das métricas TTFF (Time To First Fixation) e TTFMC (Time To First Mouse Click), pode-se observar que, para concluir o primeiro passo (chegar à página inicial do site "Óticas do Povo"), os usuários de baixo letramento 
gastaram, em média, 356 segundos, enquanto que os de alto letramento levaram 26 segundos, ou seja, estes últimos foram em média 13,6 vezes mais rápidos.

$\mathrm{Na}$ data em que foi realizada a $2^{\mathrm{a}}$ tarefa, a AOI "link patrocinado do Google", correspondia a um site concorrente ao que deveria ser pesquisado. Assim, a partir das métricas FFD (First Fixation Duration) e MCC (Mouse Click Count) foi possível observar que essa AOI teve menor tempo de atenção para os usuários de baixo letramento, que fixaram o olhar nesse link por 0,11 segundos, em média. Já os usuários de alto letramento tiveram fixações de 0,32 segundos, em média e não clicaram no link do site concorrente, optando pelo link do site esperado na tarefa. Isto poderia justificar o fato da maioria dos usuários de baixo letramento ter se confundido com os sites concorrentes, necessitando reiniciar a tarefa. Em virtude do maior tempo de fixação sobre a AOI "link patrocinado do Google", observou-se que os usuários de alto letramento não se dispersaram com o link do site concorrente e focaram melhor na tarefa.

$2^{\mathbf{0}}$ passo (Busca no site Óticas do Povo): A partir da associação das métricas TTFF (Time To First Fixation) e TTFMC (Time To First Mouse Click) com a AOI "menu lojas Óticas do Povo", pode-se afirmar que o intervalo de tempo entre a chegada à página inicial do site e o clique no menu "Lojas" foi maior para os usuários de baixo letramento (94 segundos, em média) em comparação com o intervalo dos usuários de alto letramento (02 segundos, em média). Pode-se assim, afirmar que os usuários de baixo letramento são mais suscetíveis a problemas de interface, como a falta de legibilidade do menu "Lojas", causada pelo tamanho reduzido da fonte e pelo baixo contraste de cor. Isso foi confirmado na entrevista pós-teste, quando o participante foi questionado sobre alguma sugestão de melhoria do site, apesar de já terem escrito no questionário que não teriam, mas na entrevista eles disseram que o tamanho das letras podia ser maior e que não estavam enxergando direito por causa das cores.

Uma característica que pode ter contribuído para esse tempo maior é a pouca legibilidade do menu "Lojas", que apresenta um tamanho reduzido da fonte e um baixo contraste de cor.

A partir da métrica FC (Fixation Count), observou-se que, apesar do rastreador ter registrado dezessete fixações sobre o menu "Lojas", por parte do usuário de baixo letramento LL_04, este não associou o rótulo do menu ao objetivo da tarefa. Em contrapartida, associando a métrica FC (Fixation Count) à AOI "banner inferior Óticas do Povo", correspondente a um link promocional (com imagens de óculos), este mesmo usuário fez 28 fixações nesse banner, acreditando ser o caminho para chegar aos endereços das lojas. Sua intenção ficou clara na verbalização consecutiva, quando o usuário LL_04 afirmou: - Eu estava tentando "entrar" na ótica pela foto da vitrine da loja. Os endereços deviam estar lá "dentro". Quando o usuário clicou nesse link, foi reportado para uma página de ofertas, tendo se frustrado, ainda mais, por não encontrar os endereços das lojas. Depois de cinco minutos de teste (mais da metade do tempo para realizar a tarefa), este usuário recebeu ajuda dos pesquisadores e voltou a navegar no menu horizontal superior para, finalmente, interagir com o menu "Lojas" para cumprir a tarefa. Este mesmo link promocional recebeu, em média, seis fixações dos outros quatro usuários de baixo letramento, mas foi ignorado pelos usuários do outro grupo. 


\section{Sugestões de melhoria da interface para usuários de baixo letramento}

Com o estudo, alguns comportamentos e dificuldades comuns aos usuários de baixo letramento foram percebidos:

Os rótulos usados para elementos da interface, como botões, itens de menu entre outros, devem ser objetivos e claros de modo a não induzir o usuário ao erro. Um exemplo ocorreu no botão "Pesquisa Google", que fez com que dois participantes selecionassem esse botão, antes de digitarem algo no campo de pesquisa, acreditando que só assim começariam a pesquisa no Google. Projetar interfaces com linguagem simples de modo a facilitar a navegação para esse tipo de usuário, uma vez que os mesmos não têm as habilidades de leitura e escrita de forma plena.

Como esse tipo de usuário normalmente não utiliza as ferramentas de busca dos sites comerciais, preferindo navegar pelo menu, que não devem conter muitos subitense estes devem ser rotulados de forma simples, pois o uso de palavras pouco usuais ou técnicas, pode gerar perda de tempo e desistência do usuário. É possível citar, como exemplo, a palavra "Hidráulica" presente no site da primeira tarefa. Dificilmente, o participante de baixo letramento saberá o significado dessa palavra que poderia ser substituída por "canos e água". Para o site da segunda tarefa, sugere-se a substituição do menu "Lojas" por "Endereços".

Deve-se procurar usar apenas imagens que contribuam para o entendimento das tarefas. O gazeplot mostrou que um dos participantes de baixo letramento ficou distraído com a foto de uma conhecida modelo brasileira, demorando assim mais tempo para cumprir a tarefa. Deve-se tomar cuidado com o uso de imagens de forma que elas não causem ambiguidades nem confundam os usuários. Em função do tempo e das dificuldades que os usuários de baixo letramento tiveram para concluir a segunda tarefa, sugere-se filtro de busca por ordem alfabética.

\section{Conclusão}

Esta pesquisa teve como objetivo avaliar a interação de usuários brasileiros com baixo letramento em interfaces de sistemas web, com foco nas características de acessibilidade e de usabilidade, por meio de rastreamento ocular (eyetracking). Foram analisados os comportamentos de usuários com alto e baixo letramento durante a navegação. Para isso foi utilizado o equipamento Tobii T120 para a aplicação dos testes com gravação de vídeo, além de questionários e entrevistas.

Os pesquisadores determinaram as métricas e áreas de interesse dos participantes, e após os testes, o rastreador gerou relatórios da navegação de cada usuário com um grande número de dados. Esse recurso permitiu que o trabalho tivesse uma maior riqueza de detalhes, que apenas a observação ou mesmo a filmagem do usuário não permitiriam. Os resultados do eyetracking indicaram diferenças na navegação de usuários com perfis distintos de letramento. Através das métricas do rastreamento ocular, principalmente pela quantidade e duração das fixações, foi possível mensurar o grau de dificuldade que usuários de baixo letramento têm para navegar, indicando que este perfil, lêem absolutamente todo o texto das páginas de modo a terem segurança na tomada de decisão e que as imagens, quando não contextualizadas, podem ser fatores de dispersão. As recomendações de melhoria de interface necessitam de validação, embora tenham sido embasadas por trabalhos anteriores que já foram 
mencionados na seção 2.3. Espera-se que estas sugestões possam contribuir no desenvolvimento de interfaces acessíveis a esse tipo de usuário. Recomenda-se para trabalhos futuros que seja utilizado um número maior de usuários para realizar um tratamento estatístico dos dados.

\section{Referências}

Associação Brasileira de Empresas de Pesquisa. Critério de Classificação Econômica Brasil (CCBE 2013 - Base LSE 2011). Disponível em: http://www.abep.org/new/criterioBrasil.aspx. Acessado em: 17/01/2014.

Barboza, E.M.F. A linguagem clara em conteúdos de websites governamentais para promover a acessibilidade a cidadãos com baixo nível de escolaridade. Revista do Instituto Brasileiro de Informação em Ciência e Tecnologia (2011).

Braga, H., Pereira, L. S., Ferreira, S. B. L., Silveira, D. S.: Aplicando o Método Percurso com Barreiras: indo além da avaliação automática de acessibilidade. Trabalho publicado no IX Simpósio Brasileiro de Sistemas de Informação. João Pessoa. Maio de 2013.

Capra, E. P., Ferreira, S. B. L., SILVEIRA, D. S., Modesto, D.: Evaluation of Web Accessibility: an Approach Related to Functional Illiteracy Denis Silva da Silveira e Debora Modesto- Trabalho publicado em: Procedia Computer Science - 2012 http://www.sciencedirect.com/science/journal/18770509/14 - ISSN 18770509 Volume 14 - páginas 36-46

Capra, E. P- "Protocolos de Avaliação de Acessibilidade Web comAnalfabetos Funcionais" Programa de Pós Graduação em Informática - Dissertação de Mestrado Departamento de Informática Aplicada da Universidade Federal do Estado do Rio de Janeiro (UNIRIO) - Eliane Pinheiro Capra - 22/9/2011

Ferreira, S.B.L., Nunes, R.R.: e-Usabilidade. LTC, Rio de Janeiro (2008)

Gupta, N.K., Rosé, C.P. Understanding Instructional Support Needs of Emerging Internet Users for Web based Information Seeking. Journal of Educational Data Mining 2, 2010, 38-82.

Hearst, M.A.: Search User Interfaces. Cambridge University Press, New York (2009).

Instituto Brasileiro de Geografia e Estatística: Síntese de Indicadores Sociais: Uma Análise das Condições de Vida da População Brasileira, 2010. Available at: http://www.ibge.gov.br/home/estatistica/populacao/condicaodevida/indicadoresmini mos/sinteseindicsociais2010/SIS_2010.pdf. Retrieved April 04, 2012.

Kodagoda, N., Kahan, N., Wong, W.: Identifying Information Seeking Behaviors of Low and High Literacy Users: Combined Cognitive Task Analysis. In: Proceedings of NDM9, the 9th International Conference on Naturalistic Decision Making, (The British Computer Society, London, 2009), 347-354.

Kodagoda, N., Wong, B.: Effects of Low \& High Literacy on User Performance in Information Search and Retrieval. In: Proceedings of the 22nd British HCI Group Annual Conference on People and Computers: Culture, Creativity, Interaction, pp.173-181. The British Computer Society, Swinton, UK (2008) 
Lukasova, K. Movimento ocular em crianças e adultos: estudo comparativo com uso integrado de RMf e EyeTracking. Tese de Doutorado. Faculdade de Medicina da Universidade de São Paulo. Programa de Radiologia. São Paulo, 2012.

Marshall, P., The importance of reading comprehension, No Date. Available at: http://www.k12reader.com/the-importance-of-reading-comprehension/. Retrieved: July 23, 2013.

Melo, A.; Piccolo, L.; Ávila, I.; Tambascia, C. Usabilidade, Acessibilidade e Inteligibilidade Aplicadas em Interfaces para Analfabetos, Idosos e Pessoas com Deficiência. Resultados do Workshop do VIII Simpósio Brasileiro sobre Fatores $\begin{array}{llll}\text { Humanos em } & \text { Sistemas } & \text { Computacionais. }\end{array}$ In:<http://www.cpqd.com.br/file.upload/1749021822/resultados_workshop_uai.pdf $>$. Acess: 09 jan 2014.

Modesto, D.M. and Ferreira, S.B.L. - Guidelines for search features development - a comparison between general users and users with low reading skills - Paper published in Proceedings of the 5th International Conference on Software Development for Enhancing Accessibility and Fighting Info-exclusion (DSAI 2013), - University of Vigo - Vigo - Espanha - November 2013 http://www.sciencedirect.com/science/journal/18770509/14 - ISSN 18770509

Modesto, D.M. and Ferreira, S.B.L. and Alves, A.S. - Search Engine Accessibility for Low-Literate Users - - 5th International Conference on Human-Computer Interaction Las Vegas - EUA - July 2013 - ISBN: 978-3-642-39264-1

Modesto, D.M. Acessibilidade de Recursos em uma Interface de Motor de Busca com Foco em Usuários com Baixo Letramento - Programa de Pós Graduação em Informática - Mestrado - Departamento de Informática Aplicada da Universidade Federal do Estado do Rio de Janeiro (UNIRIO) - Débora Maurmo Modesto- 09/2012

Nielsen, J., Loranger, H.: Usabilidade na Web: Projetando Websites com Qualidade. Elsevier, Rio de Janeiro 2007

Nielsen, J. Why You Only Need to Test With 5 Users, 2000b. Available at: < http://www.useit.com/alertbox/20000319.html>. Retrieved July 28, 2008.

TOBII. Available at: http://www.tobii.com/en/eye-trackingresearch/global/products/hardware/tobii-t60t120-eye-tracker/. Retrivied Dezember, 2013.

Thurow, S., Musica, N.: When Search Meets Web Usability.NewRiders,Berkeley2009

United Nations Educational, Scientific and Cultural Organization.: Understandings of Literacy. In: The Education For All Global Monitoring Report: Literacy for Life, 2006, 149-159.Available at: http://www.unesco.org/education/GMR2006/full/chapt6_eng.pdf. Retrieved April 13, 2012. 